\title{
Epilepsy Surgery in Children in Resource Poor Setting
}

\author{
Mahesh Kamate $^{1}$ - Vishaka Kothiwale ${ }^{2}$. Shivashankar Marajakke ${ }^{3}$
}

Received: 7 February 2020 / Accepted: 19 March 2020 / Published online: 1 July 2020

(C) Dr. K C Chaudhuri Foundation 2020

To the Editor: Epilepsy surgery is emerging as an important modality for treatment of drug resistant epilepsy (DRE) in children in India in the last 1-2 decades [1]. If properly chosen, epilepsy surgery provides good seizure control in almost $2 / 3$ rds of cases [2]. Advantages of epilepsy surgery include seizure freedom in majority of children and reduction of burden of anti-epileptic drugs (AEDs) in almost all children [2]. The main barrier for epilepsy surgery in children in India is the availability of the epilepsy surgery services. Currently it is available in very few government hospitals, where there is long waiting lists and in private hospitals in metropolitan cities where the cost is very high. Proper pre-surgical evaluation is very important before surgery and this needs modalities like video assisted EEG, 3-Tesla MRI, PET (positron emission tomography) scan, functional MRI, electrocorticography and stereo-EEG which are very expensive [3]. This deters many pediatricians from referring cases for surgery and many parents reject this option. However, not all cases need such detailed evaluation and many of these children can be operated with good results in resource-limited settings also. These procedures include functional hemispherotomy for hemispheric epilepsies; corpus callosotomy for refractory drop-attacks in Lennox Gastaut syndrome (LGS) and reflex epilepsy and anterior temporal lobectomy for unilateral mesial temporal sclerosis with 70-80\% seizure freedom rates [4]. Hemispheric epilepsies and LGS form a major chunk of refractory epilepsies in children in our country [5]. Unfortunately, many pediatricians, pediatric neurologists and neurosurgeons are not aware of this.

Mahesh Kamate

drmaheshkamate@gmail.com

1 Division of Pediatric Neurology, Department of Pediatrics, KAHER's Jawaharlal Nehru Medical College, Belagavi, Karnataka, India

2 Department of Pediatrics, KAHER's Jawaharlal Nehru Medical College, Belagavi, Karnataka, India

3 Department of Neurosurgery, Siddhagiri Hospital and Research Centre, Kanerimath, Kolhapur, Karnataka, India
We operated on 6 cases of DRE with good results in last 2 y with available limited resources using government schemes. Three cases with hemiparetic cerebral palsy and symptomatic epilepsy, and 1 with hemiconvulsion-hemiplegia-epilepsy syndrome underwent functional hemispherotomy; 1 with temporal lobe epilepsy underwent anterior temporal lobectomy and 1 with LGS underwent one-stage corpus callosotomy. While two patients who underwent hemispherotomy and one with temporal lobectomy are off medications after 6 mo of surgery, remaining three are maintaining on minimal AEDs (antiepileptic drugs) $12-18$ mo post-surgery. There was no worsening in the hemiparesis after surgery but in two, it actually improved. All the cases reported improvement in cognition, behavior and quality of life. In all the cases, there was a delay of 2-6 y before surgery was considered and all had failed more than 5-6 AEDs.

Epilepsy surgeries like hemispherotomies, corpus callosotomies and temporal lobectomies which are needed by many patients in developing countries can be easily done in many centers with limited resources.

\section{Compliance with Ethical Standards}

Conflict of Interest None.

\section{References}

1. Shah R, Botre A, Udani V. Trends in pediatric epilepsy surgery. Indian J Pediatr. 2015;82:277-85.

2. Rathore C, Radhakrishnan K. Epidemiology of epilepsy surgery in India. Neurol India. 2017;65:S52-9.

3. Menon RN, Radhakrishnan K. A survey of epilepsy surgery in India. Seizure. 2015;26:1-4.

4. Jayalakshmi S, Vooturi S, Gupta S, Panigrahi M. Epilepsy surgery in children. Neurol India. 2017;65:485-92.

5. Dwivedi R, Ramanujam B, Chandra PS, et al. Surgery for drugresistant epilepsy in children. N Engl J Med. 2017;377:1639-47.

Publisher's Note Springer Nature remains neutral with regard to jurisdictional claims in published maps and institutional affiliations. 